\title{
Automated Shack-Hartmann seeing measurements at the South Pole
}

\author{
T. Travouillon ${ }^{1}$, M. C. B. Ashley ${ }^{1}$, M. G. Burton ${ }^{1}$, J. W. V. Storey ${ }^{1}$, P. Conroy ${ }^{2}$, G. Hovey ${ }^{2}$, M. Jarnyk ${ }^{2}$, \\ R. Sutherland ${ }^{2}$, and R. F. Loewenstein ${ }^{3}$ \\ 1 School of Physics, University of New South Wales, Sydney, NSW, 2052, Australia \\ 2 Mount Stromlo Observatory, Australian National University, ACT, 2611, Australia \\ 3 University of Chicago, Yerkes Observatory, 373 W. Geneva Street, Williams Bay, WI 53191, USA
}

Received 2 June 2003 / Accepted 30 July 2003

\begin{abstract}
The statistics and dynamics of the atmospheric seeing at the South Pole have been studied over a period of 101 days in winter. These measurements have been made with the first fully autonomous differential image motion monitor, the A-DIMM. The analysis shows an average seeing of $1.9^{\prime \prime}$ with a standard deviation of $0.6^{\prime \prime}$. The extensive set of data has allowed the study of the seeing time variations, showing that the seeing varies by a factor of two within a characteristic time of 2 hours.
\end{abstract}

Key words. site testing - turbulence

\section{Introduction}

As part of a site testing campaign in Antarctica, the Joint Australian Centre for Astrophysical Research in Antarctica (JACARA) has deployed a seeing monitor at the South Pole station. While the median seeing of the site has already been determined using $C_{N}^{2}$ profiles (Marks et al. 1996; Marks et al. 1999; Travouillon et al. 2003), a direct, quantitative measurement of the seeing has only been carried out for a limited period of time in 1996 using a DIMM (Bally et al. 1996; Loewenstein et al. 1998). It was found that, although the South Pole is currently the world's best ground-based observation site in terms of infrared sky background, it has poor average seeing of about 1.7" in the visible. On the other hand, the vertical distribution of the atmospheric turbulence at the site is very advantageous. The entire turbulence is located within a low altitude boundary layer about $300 \mathrm{~m}$ high and the jet stream is totally absent. For this reason, the South Pole is predicted to have a larger isoplanatic angle and coherence time than temperate sites (Marks et al. 1999; Marks 2002; Travouillon et al. 2003).

In order to extend our knowledge of the statistical behaviour of the seeing conditions at the South Pole, the A-DIMM (Automated Differential Image Motion Monitor) was specially engineered to work totally autonomously in the cold Antarctic conditions, measuring the seeing in a semicontinuous manner throughout the whole Antarctic winter of 2001. The A-DIMM incorporates a Shack-Hartmann wavefront sensor which uses a microlens array to split the light entering

Send offprint requests to: $\mathrm{T}$. Travouillon, e-mail: tonyt@phys.unsw.edu.au the telescope and form a series of images spanning the whole aperture. This technique is very commonly used in adaptive optics to assess the wavefront abberation. For our site testing purposes, the A-DIMM acts as an improved DIMM, using an array of 24 sub-apertures instead of the standard double aperture, thereby obtaining multiple simultaneous measurements of the seeing.

In this paper, we present the characteristics of the A-DIMM and its mount (G-Mount). We also present the results of its first year of seeing measurements at the South Pole.

\section{System description}

The A-DIMM, like all classical DIMMs relies on the method of differential image motion between two or more sub-apertures of the instrument to calculate the seeing parameter $r_{0}$. The theory of these instruments is described in details in Roddier (1981).

Like all of the other site testing experiments deployed with the AASTO (Automated Astrophysical Site Testing Observatory) Storey et al. (1996), the A-DIMM was designed and built with three essential requirements. The telescope must be able to endure temperatures as low as $-80^{\circ} \mathrm{C}$ without losing its optical properties. It must run in a completely autonomous mode so as to minimise maintenance and allow for remote operation via satellite. It must also require only a very small amount of electrical power.

The A-DIMM (Dopita et al. 1996) was built around the optics of a $35 \mathrm{~cm}$ diameter Celestron C14. The tube of the telescope was replaced by an Invar structure in order to better 
match the coefficient of expansion of the Pyrex mirrors. This allowed the A-DIMM to retain its focus between the commissioning in Australia at $+20^{\circ} \mathrm{C}$ and the installation at the South Pole at $-30^{\circ} \mathrm{C}$. Any step at the edge of the window has been removed to discourage ice build up. The telescope can regularly park itself into heated cups to sublime any ice that could have formed on the window.

The automated Cassegrain instrument is capable of centering a star by reflecting the light with a pierced mirror onto a finder CCD camera. The information on the star position is used to offset the telescope in order to let the light pass through the pierced hole of the mirror onto the Shack-Hartmann optics. This technique is used to automatically centre the chosen star taken from a pre-selected catalogue. The catalogue consists of 48 stars with visual magnitude brighter than 3 and evenly distributed across the sky. The script used to acquire the data analysed in this paper runs for 10 hours. It starts observing the first star on the catalogue and will do so for 30 min unless the star cannot be found (because of clouds, for example). In either case, it will then move on to the second star in the script and will continue this operation until the script time is up. This allows us to maximise the observation time in case of scattered cloud obscuring parts of the sky without help of a human intervention.

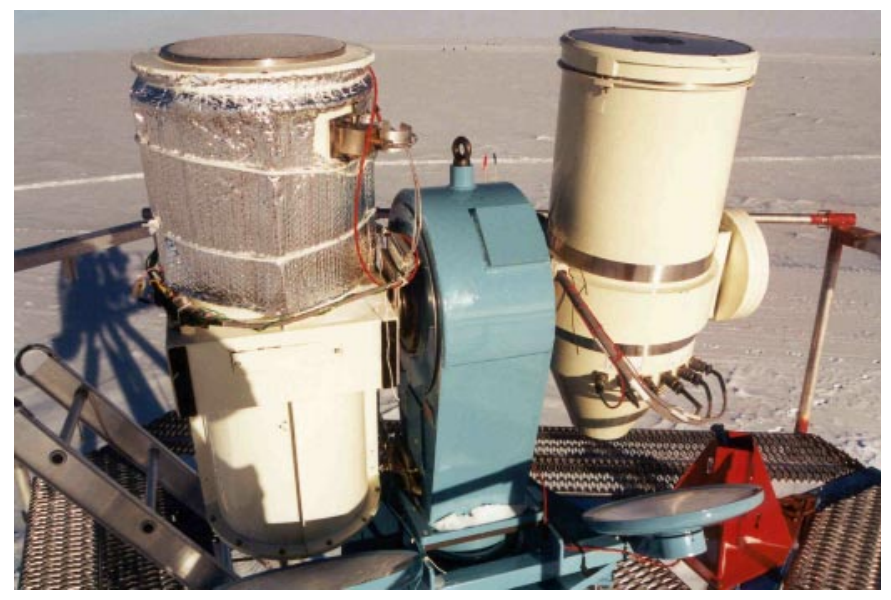

Fig. 1. A-DIMM (right) installed at the South Pole on the G-mount. The instrument on the left if the AFOS.

Once the star is detected and centered, the light is collimated to form a pupil on the surface of a microlens array. Each square lens $(0.188 \mathrm{~mm}$ on a side) forms a separate image of the target star on the CCD detector with a scale of $1.09(\mathrm{H}) \times$ 1.26(V) arc sec/pixel, corresponding to an effective $64 \mathrm{~mm}$ square at the entrance aperture of the telescope. This size is chosen to be smaller than the typical value of the expected Fried parameter (Travouillon et al. 2003; Marks et al. 1999) in the spectral range of the telescope. The A-DIMM freezes the seeing with exposure times of $10 \mathrm{~ms}$. This number was chosen to be as large as possible yet within the time range where the atmosphere retains its statistical properties $\left(t<r_{0} / v_{w}\right)$, using typical wind speed $v_{w}=5 \mathrm{~ms}^{-1}$ and $r_{0}=5 \mathrm{~cm}$.

With this technique, the A-DIMM obtains 24 images from the corresponding sub-apertures, making a total of

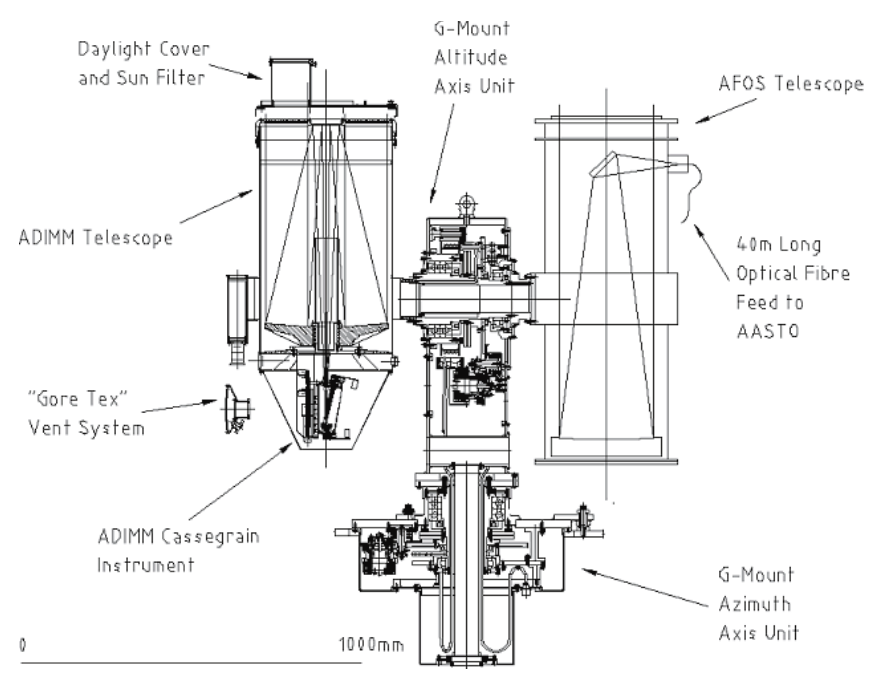

Fig. 2. Cross section through the G-mount assembly with the A-DIMM and AFOS telescopes.

215 separate baselines satisfying the condition $(d>2 D)$. Subpixel centroiding is then used on each individual image in order to determine their respective distances. The accuracy of this method will be discussed in the next section. Once the information on the coordinates and amplitude of each image is compressed and stored, the A-DIMM discards the image and repeats the observation at a rate of one image every five seconds. While the frequency of the measurements is low compared to other DIMMs, the large number of baselines improves our statistics.

The A-DIMM was installed at the South Pole station in the summer 1999. The telescope is driven by the G-Mount, an Alt/Az mount supporting two telescopes. The G-mount can be programmed to schedule the observation of the two telescopes installed. In 2000, the A-DIMM was installed aside the AFOS (Antarctic Fibre Optic Spectrometer, Boccas et al. 1998), but technical problems with the AFOS allowed $100 \%$ of the observation time to be spent with the A-DIMM during the year 2001. The overall assembly is showed in Fig. 2. The G-mount was installed on top on a $7.5 \mathrm{~m}$ high hexapod tower which, in theory, retains its parallelism with the ground under any wind conditions.

\section{Data processing}

The data are partially processed by the A-DIMM itself in order to reduce the bandwidth needed to send the data outside the Antarctic. The DIMM's software calculates the centre of mass of each of the 24 sub-aperture images that forms within a 9 pixel square. The centroid coordinates and fluxes are recorded and compressed before the image is discarded. Every $5 \mathrm{~s}$ such an image is taken and the resulting information (using 322 bytes of disk space) is appended to the daily file.

The differential image motion is calculated by measuring the variance of the distance between two sub-aperture images. As seen in Sect. 2, each baseline gives 2 estimates of the seeing. Every 5 seconds, the 215 baselines therefore measure 430 separate distances. The variance is then calculated over 
25 successive measurements. The error analysis and the statistical treatment of the data are now reviewed in detail.

\subsection{Instrumental noise}

The differential nature of DIMM's operating mode means that the measurements are unaffected by vibrations of the telescope and tracking errors. This is a major advantage as it eliminates most sources of errors. However, as described in Sarazin et al. (1990) and Tokovinin (2002), subtle effects must be taken into account to remove potential bias in the measurement of the seeing.

The first source of bias is the accuracy of the centroiding. Under a zero turbulence condition, the centroid position will vary due to photon noise and algorithm error. This instrumental noise will appear as a variance $\sigma_{\text {inst }}^{2}$ which will be assumed to be systematic and isotropic. The instrumental variance must be subtracted out from the total measured variance in order to obtain the turbulence generated variance:

$\sigma_{\text {tur }}^{2}=\sigma_{\text {meas }}^{2}-\sigma_{\text {inst }}^{2}$

The instrumental noise was evaluated from a series of short observations of the binary star system alpha centauri. Since the seeing has been taken out by the short exposure, the change of distance between the two stars within a sub-aperture can only be due to the instrumental noise. The distance between the two stars within each sub-aperture was calculated using the same algorithm used for the centroiding. The variance of this distance was then computed and averaged using all 24 apertures. Under these conditions, we found that:

$\sigma_{\text {inst }}^{2}=0.0740^{\prime \prime} \pm 0.0008$.

This value is expected to remain the same for all stars observed, as they have been chosen to be bright enough to have a negligible photon noise level. In poor seeing conditions, the development of speckles and scintillation can increase the centroiding noise. This effect will however be small compared to the resulting variance in the seeing between all the baselines.

\subsection{Statistical errors}

In a case of multiple baseline measurements, the statistical error is derived from the standard deviation that results from the calculation of the seeing at each individual independent baseline. In good seeing conditions, the centroiding of the single speckle will be done accurately. When the seeing is poor $\left(>2^{\prime \prime}\right)$ the star breaks up in multiple speckles and each baseline measures a different centroid. Each seeing measurement will therefore carry a variable error, dependent in a complicated way on the seeing value instead of a constant percentage error for the single baseline DIMMs (Sarazin et al. 1990).

The error found with the A-DIMM at the South Pole is on average $17 \%$, a value larger than the typical single aperture DIMM $(\approx 10 \%)$. This can be explained by the larger seeing values measured at the South Pole station, as will be shown in the next section.

\section{Results}

\subsection{Results statistics}

The data presented in this paper represent an extensive coverage of the seeing conditions at the South Pole Station. Spanning the major part of the winter night, between the 28 May and the 6 September 2001, 588 individual measurements have been made. A statistical summary of the results is presented in Table 1 and is compared to other measurements of the seeing at the site.

The A-DIMM measurements confirm and extend the known seeing characteristics of the site. Although the turbulence is known to be concentrated in a thin boundary layer, the resulting ground level seeing is of poor quality. The seeing drops below $1^{\prime \prime}$ for only $10 \%$ of the time. It is noted that the A-DIMM shows a higher average seeing than the H-DIMM or the microthermals. This can be explained by the autonomous nature of its operation. The H-DIMM and microthermal data were taken manually and therefore do not include the more extreme conditions where no same person would go outside and physically observe. In such conditions (strong wind, hazy sky) the A-DIMM continues to take data although they are likely representative of bad seeing. Figure 3 shows the seeing distribution.

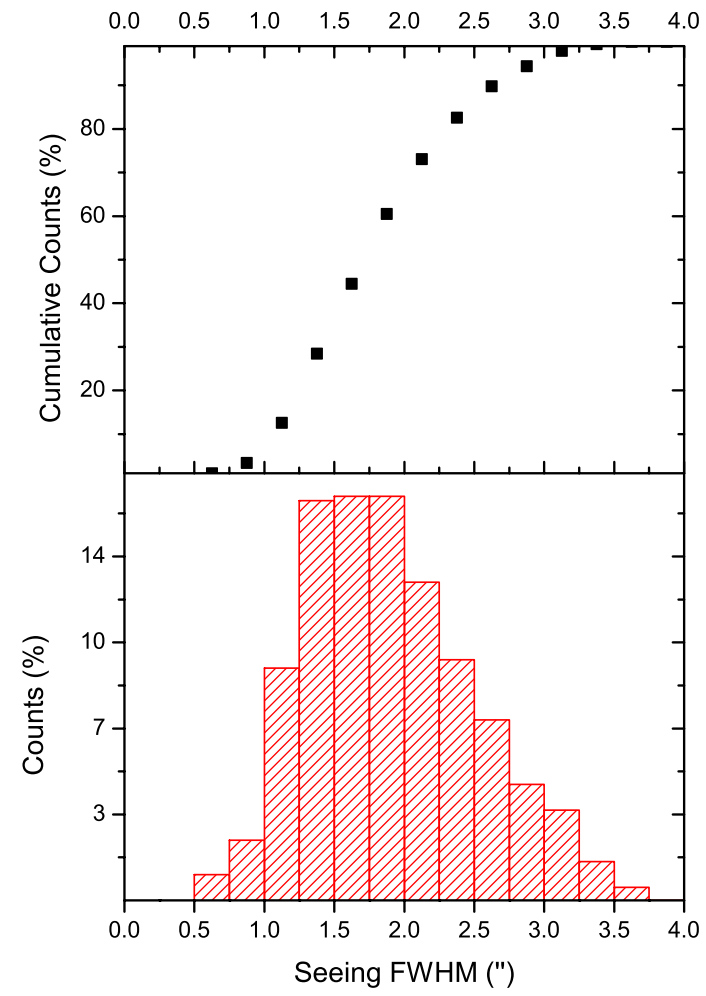

Fig. 3. Simple and cumulative seeing distribution at $500 \mathrm{~nm}$.

The statistical distribution of the A-DIMM results is in excellent agreement with the H-DIMM (Loewenstein et al. 1998) seeing probability. The only noticeable difference between the two data sets comes from the worst seeing measured. The A-DIMM worst seeing is $3.72^{\prime \prime}$ while the H-DIMM recorded seeing as poor as 6.2". We believe that this 
Table 1. South Pole seeing as measured by the A-DIMM in 2001 at a wavelength of $500 \mathrm{~nm}$. It is compared to other seeing measurements done at the South Pole. All values are in arcseconds.

\begin{tabular}{llllllll}
\hline \hline Instrument & Sample size (days) & Mean & Std. Dev. & Median & Best 25\% & Best & Worst \\
\hline A-DIMM (This work) & 101 & 1.90 & 0.60 & 1.82 & 1.43 & 0.46 & 3.72 \\
SODAR (Travouillon et al. 2003) & 269 & 1.73 & 1.07 & 1.59 & 1.17 & $<0.3$ & 8.11 \\
Microthermals (Marks et al. 1999) & 16 & 1.86 & 0.75 & 1.6 & 1.0 & 0.8 & 3.1 \\
H-DIMM (Loewenstein et al. 1998) & 28 & 1.53 & - & 1.64 & - & 0.6 & 6.2 \\
\hline
\end{tabular}

difference comes from the lower sampling speed of the ADIMM. Extremely high seeing values last for a very short period of time and can therefore be missed by the A-DIMM.

\subsection{Temporal variations}

Looking at the long-term fluctuations of the seeing (Fig. 4), no conclusion can be made about a seasonal dependence of the seeing. The month of July is the most stable, as was also noticed the year before (2000) by Travouillon at al. The daily average seeing varies between $1.5^{\prime \prime}$ and $2^{\prime \prime}$ during this period while in June and August there are variations of up to $2^{\prime \prime}$ over a few days.

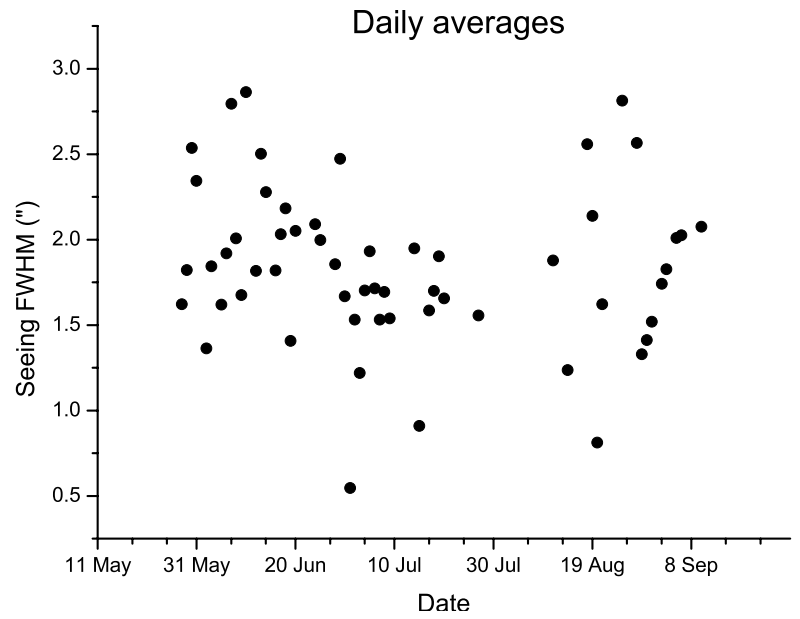

Fig. 4. Entire data sample summarised in daily averages.

The short term temporal behaviour, has not been studied at the South Pole before. In fact, although such data are commonly recorded at observatories, temporal behaviour has only been, to our knowledge, the subject of two other papers. Racine 1996 and Vernin et al. (1998) studied this characteristic at Mauna Kea and at the observatory of the Roque de los Muchachos respectively. The scientific motivation behind this information is the management of of the observatory schedules. Many observatories now offer a queue scheduling which prioritorises certain science projects for windows of particularly good seeing. The duration of these windows and the level of variation are therefore other figures of merit that can be associated with a site.

To allow direct comparison, we have followed the technique developed by Racine (1996). We introduce the temporal structure function which measures the fractional change of the seeing over a time period $\Delta t$ :

$f(\Delta t)=\frac{|\varepsilon(t+\Delta t)-\varepsilon(t)|}{\varepsilon(t+\Delta t)-\varepsilon(t)}$.

This expression is related to the seeing ratio over an interval time $\Delta t$ by:

$X(\Delta t)=\left[\frac{\varepsilon(t+\Delta t)}{\varepsilon(t)}\right]^{\operatorname{sign}(\varepsilon(t+\Delta t)-\varepsilon(t))}=\frac{1+f(\Delta t)}{1-f(\Delta t)}$.

Provided that the seeing distribution is log-normal (Vernin et al. 1998), this expression should saturate at $\langle f(\infty)>$ within a characteristic time $\tau$ and at a growth rate $\gamma$. The temporal structure function can be represented by the relation:

$<f(\Delta t)>=<f(\infty)>\cdot[1-\exp (-\Delta t / \tau) \gamma]$.

We have chosen to estimate the average temporal structure function using the data from a 5 day period where the seeing was available continuously for 4 hours at a time at intervals of $5 \pm 1 \mathrm{~min}$. Each string was analysed in a manner similar to Racine (1996) and then averaged. The data were finally fitted to Eq. (5) with $\chi^{2}$ minimisation. The fitting (see Fig. 5) yields:

$\left\{\begin{array}{l}<f(\infty)>=0.33 \pm 0.03 \\ \tau=130 \pm 50 \mathrm{~min} \\ \gamma=0.70 \pm 0.08\end{array}\right.$

These results show that the seeing dispersion saturates between one and three hours to a seeing ratio $X(<f(\infty)>)=1.96$. Racine (1996) observed a ratio of 1.56 in a characteristic time of 17 minutes at Mauna Kea. The South Pole seeing therefore varies more slowly but has larger amplitudes. In fact the dispersion time at the South Pole is comparable to that measured by Vernin et al. 1998 using a different mathematical approach at Roque de los Muchachos (1.2 hours), although the average seeing at that site is lower $\left(\simeq 0.6^{\prime \prime}\right)$.

Figure 5 shows a large scatter due to the small amount of data averaged. This scatter is also present in the data of Racine (1996) and Vernin et al. (1998). A component of this scatter may be quasi-periodic and previous authors have suggested that it may arise from gravity-driven atmospheric waves, as their period matches the period of seeing dispersion. As described previously, the South Pole seeing is produced by turbulence in a boundary layer $300 \mathrm{~m}$ thick. Gravity waves therefore cannot explain the variations at the South Pole. Instead, we believe that the sharp boundary layer causes shear instability. This statement is supported by Travouillon et al. (2003) who found that 


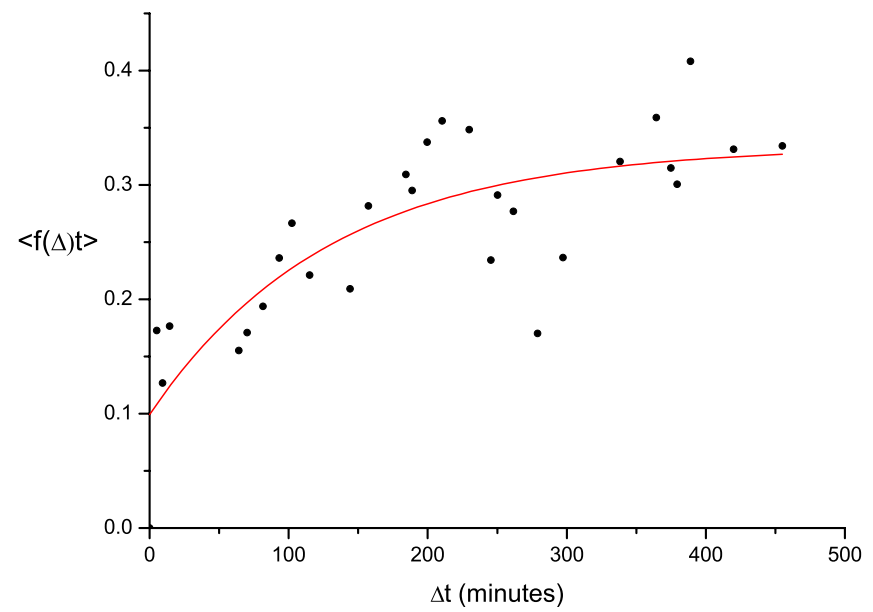

Fig. 5. Average seeing dispersion as a function of time. The line follows Eq. (5), minimising $\chi^{2}$.

the boundary layer turbulence was strongly correlated with vertical wind speeds. The periodicity of the seeing might therefore follow the frequency of the instability waves created at the boundary between the two air masses near the ground. To investigate this supposition further, high spatial and time resolution profiles of the temperature in the low atmosphere would be required.

\section{Conclusion}

In its first year of measurements, the A-DIMM has successfully operated in an autonomous mode for a whole winter. This demonstrates that optical astronomical instrumentation can be engineered to function successfully in the extreme conditions of Antarctica without the need for constant human intervention. In 2002 the telescope continued to measure the seeing in order to obtain further statistics about the site.

So far, the results have confirmed the poor quality of the ground level seeing. Large improvements can be expected from elevated telescopes or adaptive optics (Lawrence 2003).
Acknowledgements. This research was supported by grants from the Australian Research Council (ARC) and the Australian Antarctic Division. Logistic and infrastructure support were supplied by CARA and the NSF. We also would like thank Jean Vernin for helpful discussions. We particularly thank the CARA winterover scientist, Charlie Kaminski, for his outstanding support.

\section{References}

Bally, J., Theil, D., Billawala, Y., et al. 1996, Publ. Astron. Soc, Aus., $13,22-7$

Beckmann, P. 1967, Probability in Communication Engineering (New York: Harcourt, Brace, \& Word)

Boccas, M., Ashley, M. C. B., Phillips, A., Schinckel, A., \& Storey, J. W. V. 1998, PASP, 110, 306

Dopita, M. A., Wood, P. R., \& Hovey, G. R. 1996, PASP, 13, 39

Fried, D. L. 1965, J. Opt. Soc. Am., 55, 1427

Fried, D. L. 1975, Radio Science, 10-1, 71

Lawrence, J. S., Ashley, M. C. B., Burton, M. G., et al. 2002, PASA, 19 no. 3, 328-336

Lawrence, J. S. J. 2003, Opt. Soc. Am., submitted

Loewenstein, R. F., Bero, C., Lloyd, J., et al. 1998, ASP Conf. Ser., 141, 296

Marks, R. D., Vernin, J., Azouit, M., et al. 1996, A\&A, 118, 385

Marks, R. D., Vernin, J., Azouit, M., Manigault, J. F., \& Clevelin, C. 1999, A\&AS, 134, 161

Marks, R. D. 2002, A\&A, 385, 328

Phillips, A., Burton, M. G., Ashley, M. C. B., et al. 1999, ApJ, 527, 1009

Racine, R. 1996, PASP, 108, 372

Roddier, F. 1981, in Progress in Optics, ed. E. Wolff, 19, 283

Sarazin, M., \& Roddier, F. 1990, A\&A, 227, 294

Storey, J. W. V., Ashley, M. C. B., \& Burton, M. G. 1996, PASA, 13, 35

Tatarski, V. I. 1971, The effect of Turbulent Atmosphere on wave propagation, I.P.S.T, Jerusalem

Tokovinin, A. 2002, PASP, 114, 1156

Travouillon, T., Ashley, M. C. B., Burton, M. G., \& Storey, J. W. V. 2003, A\&A, 400, 1163

Vernin, J., \& Muñoz-Tuñón, C. 1995, PASP, 107, 265

Vernin, J., \& Muñoz-Tuñón, C. 1998, New Astron. Rev., 42, 451 